\title{
Vestibular rehabilitation after unilateral neuritis in the course of neuroborreliosis - a case report
}

\author{
Sylwia Krasowska',A-B,D \\ ${ }^{1}$ Aurifon Audiological Diagnostics and Therapy Centre, Lublin, Poland \\ A - Research concept and design, B - Collection and/or assembly of data, C - Data analysis and interpretation, \\ $D$ - Writing the article, E - Critical revision of the article, F- Final approval of article
}

Krasowska S. Vestibular rehabilitation after unilateral neuritis in the course of neuroborreliosis - a case report. J Pre Clin Clin Res. 2020; 14(3): 85-89. doi: $10.26444 / j p c c r / 126529$

\begin{abstract}
Inflammation of the vestibular nerve (vestibular neuronitis, VN) is the third most common reason of peripheral vertigo.Many VN reasons lead to a thorough approach to the patient and diagnostics based on excluding even the rarest causes. Such a cause may be infection with Borrelia burgdorferi, which, due to high tropism to the nervous system, damages the sheath of nerve cells, causing inflammation. The acute course of the disease provokes the use of standard treatment in the form of pharmacotherapy e.g.: with neuroleptics, anxiolytics and then beta-blockers, betahistine. The literature, however, clearly indicates the limitations of pharmacology, which weaken the vestibular compensation, prompting the rapid discontinuation of drugs and the commencement of rehabilitation. This publication presents a case report of a 37-year-old female patient diagnosed with VN and Lyme disease. The case is to present and analyze the progress of vestibular rehabilitation along with the accompanying causal treatment.
\end{abstract}

\section{Key words}

Lyme disease, Borrelia burgdorferi, rehabilitation, Unilateral prolapse of the labyrinthine function, vestibular neuritis, vestibular compensation

\section{INTRODUCTION}

Unilateral peripheral vestibular insufficiency (hyporeflexia or areflexia of the labyrinth) is a disorder that affects one ear and is not related to a dysfunction of the central equilibrium system (def. Hiller and McDonnell, 2011) [1]. The causes of peripheral and unilateral atrial damage include: perilymphatic fistula, acoustic neuroma, vestibular neuronitis (VN), labyrinthitis, Meniere's disease, and atriocochlear otosclerosis. One of the most common one-sided labyrinth problems is VN. The most likely cause of inflammation of the vestibular nerve is acute inflammation caused by infections (viral, bacterial, parasitic), immune disorders or circulatory disorders. People between 30 and 55 years of age are at risk. The disease is not accompanied by hearing damage although there may be tinnitus. There is sudden, severe vertigo (vertigo systemic, true vertigo) which increases over several hours and lasts up to several weeks. Damage to the atrium (mechanoreceptor of equilibrium system), so-called 'vestibular shock' is characterized by vegetative symptoms, such as vomiting, nausea, tachycardia, pressure surges, perceptual, postural and oculomotor syndrome. The postural syndrome consists of the inability to remain upright, tilting the body and head towards the affected side, and postural instability during movement. The perceptual syndrome includes dizziness with a subjective vertical deviation in space.

Oculomotor syndrome is a constant directional spontaneous nystagmus horizontal or horizontally-rotating, directed with the rapid phase towards the healthy ear and eye cyclotorsia. In the course of the vestibular neuritis, there is a sudden functional reorganization, accompanied by a partial or complete loss of the function of one labyrinth, and simultaneous hyperactivation of the other labyrinth (hence

Address for correspondence: Sylwia Krasowska, Aurifon Audiological Diagnostics and Therapy Center, Lublin, Poland

E-mail: sylwiabanas@wp.pl

Received: 05.08.2020; accepted:19.08.2020; first published: 04.09.2020 paralytic nystagmus towards the healthy ear and a deviation in posture towards the affected ear) [2]. After the initial period (acute phase) there is a period of symptom reduction, followed by static compensation (disappearance of vegetative symptoms) and after a few weeks, also dynamic compensation (improvement of VOR - vestibulo-ocular reflex). Although the efficiency of the labyrinth may never return to normal functioning (which is observed in videonystagmography studies), patients may not feel any symptoms [3]. After the acute phase subsides (and often during it), rehabilitation should be started in order to recover as quickly as possible.

In recent years, there has been great progress in the rehabilitation of peripheral vertigo and imbalance or persistent postural-perceptual dizziness (PPPD), as well as centrally-specific?, such as atrial migraine and multiple sclerosis (Latin: sclerosis multiplet-SM). The development of new methods of rehabilitation, especially by introducing virtual reality training, as well as increasing the awareness of clinicians as to the effectiveness of vestibular rehabilitation and non-pharmacological methods, makes patient recovery much faster. The recommendations of the American Physical Therapy Association (APTA) indicate a high degree of response to rehabilitation, both among patients with defined causes of vertigo and balance disorders, and among patients with undefined etiology. It should also be noted that the recommendation also applies to patients with Meniere's disease, for whom it is recommended to conduct therapy between attacks. APTA indicates an extremely important feature of the rehabilitation of the equilibrium system: it is safe, improves the quality of life, and significantly reduces the risk of falling, whatever the cause of the disease [4].

The principle of rehabilitation is based on a physiological phenomenon called vestibular compensation. This process takes place at the level of the central nervous system and is based on three mechanisms: adaptation, substitution and habituation. Adaptation consists in the neuronal regulation of the vestibulo-ocular reflex (VOR), the task of chich is to 
stabilize the image on the retina during head movement. The VOR is responsible for the associated eye movements in the opposite direction to the head movement. The information about the movement comes directly from the receptor of the ampullar organ of the unilateral semicircular canal (for rotational movement), and from the sacullus and untricule (for linear acceleration). This allows the patient to see binocular objects on which the eyes are focused despite the movement of the head [5].

Precht et al. were the first to describe vestibular shock following a unilateral loss of labyrinthine function as a result of a severe imbalance in the resting activity of neurons of the vestibular nucleus of the brainstem. This imbalance compensates the work of the hyperactive (healthy side) by the descending inhibitory mechanism of the olive-cochlear nucleus (Rasmussen's bundle). Especially in the case of VOR, the inhibitory mechanism of the medial and superior vestibular nuclei plays a huge role in adaptive compensation.

In the course of natural vestibular rehabilitation, neuronal regulation of the vestibulo-ocular reflex (VOR) occurs in a process called adaptation [6]. This process takes place approximately 3-4 weeks after the unilateral damage to the labyrinth. Although vertigo is reduced or resolved in adaptation, VOR remains weakened suggesting that adaptation is not sufficient for a complete reorganization of the equilibrium system. The mechanism of central substitution is then activated. This consists mainly in replacing the damaged sensory representation of the vestibular system with a different domain - prioreceptive or visual, integrating stimuli of the equilibrium system at the behavioural, cognitive and sensory level. As balance is mainly based on visual data, priority is given to enhancing visual substitution. It Has only only been recognized in recent years that the vestibular system also contributes to the formation of higher cognitive processes, including the perception of the body in space, navigation, attention, memory, mental image, and even social cognition, which is caused by the cortical projection of the vestibular system. Therefore, in the case of the uncompensated functions of the equilibrium system, the plasticity of the brain plays a huge role, thanks to which the system returns to efficiency although it requires a longer perspective. While static compensation is a rapid vestibulocentral process, dynamic recovery is a long-term distributed process that requires cognitive-behavioural activity. It is also often inhibited by factors slowing recovery, such as coexisting disease, age, gender of the patient, as well as inflammatory processes, e.g. Lyme disease [2].

This study presents the case report of a patient undergoing rehabilitation with a sudden loss of the labyrinth function, with vertigo and imbalance in the course of neuroborreliosis.

\section{CASE REPORT}

A 37-year-old female patient was urgently admitted to the neurology department with severe vertigo, nausea and intense vomiting. To-date she had been healthy with no history of chronic diseases. Neurological examination revealed no disorders of a central nature - without paresis and cerebellar symptoms. On the other hand, nystagmus was found when she looked to the left. A computed tomography (CT) scan of the head was performed, which showed no focal changes within the brain. Ultrasound (US) Doppler examination of the carotid and vertebral arteries showed no abnormalities in the morphological image and flow within the examined vessels. Blood counts showed slightly elevated levels of neutrophils and decreased levels of lymphocytes. The patient received Polvertic, Hydrochlorothiazidum, Metamizole, and Metoclopramidum in the ward. After acute dizziness subsided, she was discharged home for outpatient treatment at an audiology clinic. In an outpatient setting, three weeks after the onset of symptoms, a videonystagmographic examination (VNG) was performed which revealed uncompensated right labyrinthine areflexia in the course of VN. Spontaneous nystagmus to the left and gaze to the left were present. Reduced vestibulo-ocular reflex with a significant left-sided predominance, correct fixation, weakened vestibulo-ocular reflex and normal cervico-ocular reflex. In the FitzgeraldHallpike two-calorie test, the right labyrinth was non-reactive to a thermal stimulus (Fig. 1).
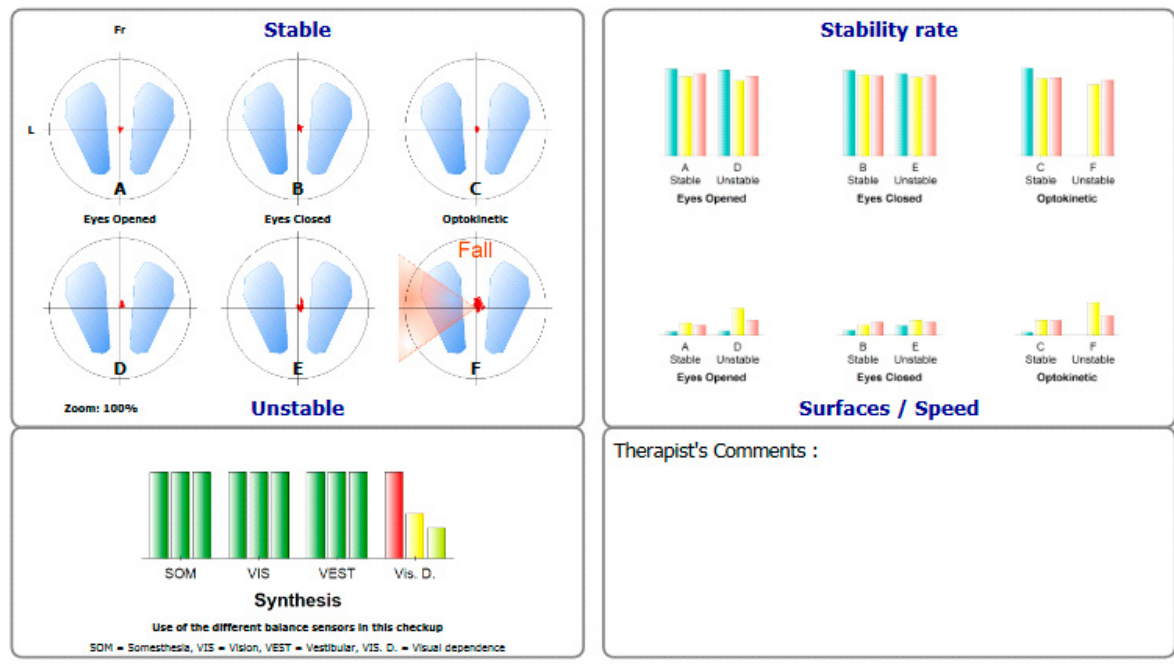

Figure 1. Fitzgerald-Hallpike bithermal caloric test indicating uncompensated one-sided prolapse of the labyrinth function (78\% reduced right-sided reflectivity). During the examination, the introduction of warm and cold air warms / cools the ear canal and middle ear. This temperature change is transferred to the horizontal semicircular canal and to the atrium by the air in the middle ear space. The endolymph closest to the channel wall is heated / cooled, making it less / denser. The heated endolymph rises and is replaced by a denser / cooler endolymph. The endolymph moves in the semicircular canal, which causes bending of the stereocilia and increased nervous stimulation. 
A dynamic posturography was also performed which indicated a significant dependence of visual inspection on the stability of the system. Additionally, the test for tonal audiometry and impedance audiometry was repeated, with no abnormal result. The patient underwent intensive compressive rehabilitation on the posturographic platform. After several weeks of training, no significant improvement was shown, which influenced the treatment team to reassess the patient's health, including performing a magnetic resonance imaging (MRI) scan of the head and neck (mainly due to neck pain and stiffness), and a screening test for $\operatorname{IgM}$ and IgG antibodies. IgM against Borrelia burgdorferi sensu Lato complex by immunoenzymatic ELISA method. The MRI showed no focal lesions, visible segments of the trigeminal, facial, vestibulocochlear nerves were without any pathological changes. The Elisa test showed a significantly increased level of IgM antibodies $>175 \mathrm{Ru} / \mathrm{ml}$. The result was confirmed by Western-Blot test - positive IgM in the range of $\mathrm{C}$ reactive protein $(\mathrm{OspC})$ for Borrelia afzelii, burgdorferi, garinii, and spielmanii. Targeted antibiotic therapy in the form of doxycyclinum $2 \times 100 \mathrm{mg}$ for 3 weeks was introduced followed by tinidazolum $2 \times 500 \mathrm{mg}$ for three days with another administration after two weeks, $2 \times 500 \mathrm{mg} / 3$ days, and rifampicin $2 \times 300 \mathrm{mg}$ for the next six weeks. At the same time, dynamic compensation therapy was carried out on the posturographic platform without changing the procedure, achieving much better results than the previous antibiotic therapy.

Six months after the onset of symptoms, the VNG test was performed again in which there was still a slight horizontal nystagmus directed with the rapid phase to the left. However, there was no nystagmus from the change of position or during the Dix-Hallpike manoeuver. The vestibulo-ocular and vestibulo-oculomotor reflexes were within the normal range. The Fitzgerald-Hallpike bi-caloric test showed slight right-sided paresis $27 \%$, compensated. The posturography test was also performed again, which showed no deviations from the norm, as shown by the combined test result before and after rehabilitation (Fig. 2).
After 80 therapeutic sessions, the patient completed her rehabilitation while still taking antibiotics. Minor imbalances and instability persisted, especially with sudden head movements.

\section{DISCUSSION}

The case presented above demonstrates otoneurological symptoms in the form of unilateral labyrinthine hyporeflexia (VN) in the course of Lyme disease, a tick-borne disease caused by Borrelia burgdorferi (B.b.) transmitted by Ixodes ricinus ticks, which is spreading increasingly rapidly in Europe and North America. Lyme disease occurs most often with coinfections, Bartonella henseale and Bartonella quaintana are the most common bacteria. The pathogens mentioned above are the most common cause of otolaryngological symptoms, which include tinnitus, dizziness, balance disorders and unilateral sensorineural hearing loss. A study by K. Sowula et al. at the Jagiellonian University in Kraków on 216 patients showed that over $50 \%$ (53.7\%) of patients in the course of Lyme disease, and Bartonella co-infection manifested symptoms in the form of dizziness and balance disorders [7]. In turn, in the Moscatello observation carried out on 266 patients diagnosed with Lyme disease, otolaryngological symptoms, including dizziness and imbalance, appeared in $11 \%$ of people. Such a large percentage of patients presenting with dizziness in the course of Lyme disease indicates the need to always consider the need to test for tick-borne diseases, especially in the case of otolaryngological symptoms which do not improve after standard treatment.

Otolaryngological manifestations in Lyme disease (specifically in Lyme disease) and co-infections can appear at any of the three stages of the disease. In another division accepted by the American Center for Disease Control and Prevention, there are two stages of Lyme disease development: early and late [8], but for the purposes of this article, three stages are distinguished. In the first phase, the most common early presentation of the disease is erythema migrans

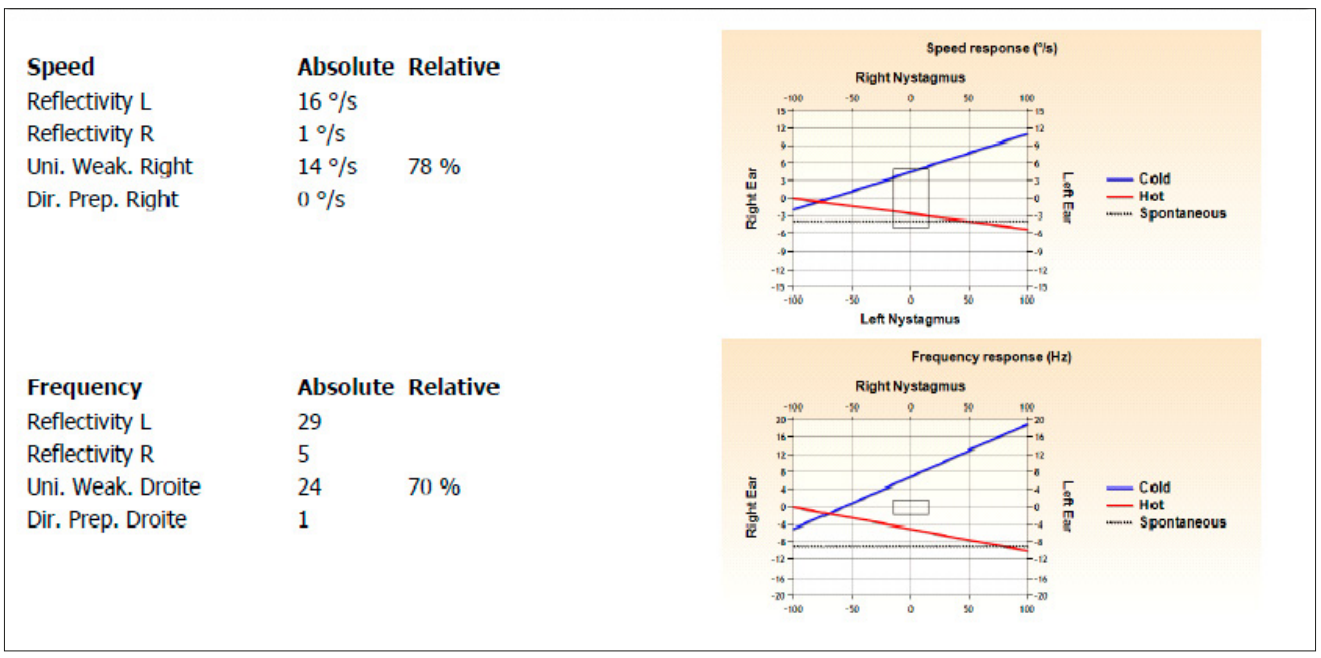

Figure 2. Comparative result of posturographic examination of a patient with unilateral loss of labyrinth function. The study shows a diminishing visual (Vis. D.) dependence of the equilibrium system. The test is an objective technique for measuring posture strategy under static and dynamic conditions, which is carried out by creates a conflicting sensory experience. The sensory organization test (SOT) on the device determines how to use the sensory systems (somatosensory, visual and vestibular) responsible for posture control. Somatosensory and visual inputs are altered by a calibrated rocking reference support surface and visual environment that moves antero-posteriorly in response to the sway of the subject's stance. The Motor Control Test (MCT) challenges posture control, causing unexpected postural disturbances 
(EM) which occurs in $60-80 \%$ of cases [9]. However, early presentations may be completely unnoticed or non-specific. It may also prezent with flu-like symptoms, such as fever, tiredness, headaches, muscle and joint pain. A few weeks or even months after infection, the second phase of the disease appears, which is already a disseminated process in chich, through blood and lymphatic vessels, as well as through the nerves, spirochetes and their products, they reach various organs causing much multi-organ damage. Assessment of the multiplicity of the immune response depends on knowledge of the diversity of the OspC antigen protein, which is an important determinant of borrelia's virulence. It has been shown, inter alia, that individual OspC recombinants induce a specific immune response, and in the presence of a specific surface antigen may influence the selection of a host for a particular strain of B.b. [10].

The biology of the selection of the humoral response exceeds the scope of this article, although it should be noted that it is not insignificant for the resulting reactions of the organism to the pathogen, because the tropism of bacteria to find $\mathrm{N}$-acetyl glucosamine (present in the connective tissue) is necessary for their growth and antigen production. Neurotropism of the bacterium B.b. in turn it induces an immune reaction which, in addition to causing damage to nervous tissue, also activates $\mathrm{T}$ and $\mathrm{B}$-dependent lymphocytes, provoking an autoreactive response against endogenous neural structures. Inflammatory changes in neurons and vessels surrounding nerves cause axonal damage and then peripheral neuropathy [11].

In studies on tick-borne infections E.Nyarko points to co-infection with Anaplasma phagocytophilum as a factor causing B.b. across the blood-brain barrier and thus increases the activity of Lyme disease in the nervous system [12]. Although, the patient has not been studied for co-infections it can be suggested that the very rapid build-up of symptoms may also be due to the presence of other tick-borne pathogens.

$5 \%$ of people with Lyme disease develop late disease presentations affecting the central and peripheral nervous systems (in the third, chronic stage of the disease several months or years after infection with Lyme disease). In the case of lesions of the peripheral nervous system, peripheral neuropathies, diffuse, heterogeneous sensory symptoms, such as paresthesia and hypoesthesia, prevail. Chronic encephalitis described by Ackermann in 1985 may also occur. Patients presented disorders of varying severity, such as spastic paresthesia, ataxia, paralysis of cranial nerves, deafness, bladder dysfunction, and cognitive impairment [13].

The diagnosis of Lyme disease is based on a two-stage serological assessment. The first stage consists in detecting IgM and IgG antibodies by ELISA and confirming it with an immunoblot test (Western blot), also in both classes. In the early phase of the disease, antibodies are not detectable in any of the classes, even in the presence of erythema migrans (EM). Therefore it is assumed that in the presence of EM, no test is performed at all before starting antibiotic therapy. In other cases, after a tick bite, the test should be perfomed a few weeks later. If neuroborreliosis is suspected, it is also advisable to test the cerebrospinal fluid in which pleocytosis is found. Although the lumbar puncture with CSF collection is the gold standard in the study of Lyme disease, it does not provide certainty as to the diagnosis and is not used in all cases [14]. In the described example, the patient did not have a cerebrospinal fluid test, but the standard two-step assessment was maintained and the symptoms suggested treatment against LNB.

A controversial question remains: did Lyme disease in the above example cause inflammation of the vestibular nerve? Józefowicz-Korczyńska et al., attempted to answer this question by reviewing publications related to balance disorders and dizziness in the course of Lyme disease. They showed that in the case of a patient with vestibular symptoms with a positive two-stage Lyme test, in accordance with the guidelines of the European Federation of Neurological Societies, antibiotics should be administered orally or intravenously and it is considered that the presented case study dealit with neuroborreliosis [15].

The aspect of slowing down the vestibular compensation process in a given patient should also be discussed. It seems that in the course of neuroborrelios, the adaptive abilities of the equilibrium system decrease, and thus the effects do not bring the desired result, even with well-chosen rehabilitation. The patient underwent a fairly quick adaptive correction, which according to many studies on vestibular rehabilitation, is the most beneficial in the process of recovering the functional efficiency of the equilibrium system. The exercises were used to improve visual-somatosensory, visual-spatial functions, behavioural-dual task exercises, kinetic enhancement of the vestibulo-ocular reflex, and sensory integration. Correctly selected exercises increased the efficiency and improved the results of objective tests; however, during the rehabilitation process, numerous decompositions appeared which spontaneously resolved in the course ofthe already treated Lyme disease. It may be suggested that the inflammation caused by the bacteria during the administration of the antibiotic generated a series of irritation reactions to the vestibular nerve [16]. Vestibular nerve involvement in the course of bacterial infection B.b. was described in 1988 by Rosenhall, in 1989 by Halperin, in 1993 by Ishizaki,althpough already in the 1920s, cases of people with cranial nerve palsy were described, with the facial nerve being most frequently affected ( $1 / 3$ cases). Peripheral paralysis of other nerves is less frequent, but infection with B.b. cannot be ruled out in the course of neurological diseases, especially those related to peripheral nerves [10].

It is recommended that in the event of a sudden loss of the labyrinth function, even with an aggressive course, support through rehabilitation should be implemented as soon as possible. M. Lacour's research on the plasticity of the brain in the process of vestibular compensation has shown that there is a 'plastic window' in which supporting compensation produces the best results, and the peak of the regulation process occurs three days after the injury. In the case of peripheral labyrinth disorders, the sooner the training is started, the better the effect [17]. Lacour also conducted a series of studies on the process of compensation and rehabilitation support. He outlined 10 rules of proper compensation training in which he included the most important strategies. Briefly, they can be presented as an individual process selected for each patient bringing together all methods of compensation, with adaptation and substitution as the most important, and with a smaller role of habituation, and importantly involving mental mechanisms in behavioural and/or cognitive therapy with the activation of motivational processes [18]. 


\section{REFERENCES}

1.McDonnell MN, Hillier SL. Vestibular rehabilitation for unilateral peripheral vestibular dysfunction. Cochrane Database Syst Rev. 2015 Jan 13; 1. https://doi.org/10.1002/14651858.CD005397.pub4.

2. Deveze A, Bernard-Demanze L, Xavier F, et al. Vestibular compensation and vestibular rehabilitation. Current concepts and new trends. Neurophysiol Clin. 2014 Jan; 44(1): 49-57. https://doi.org/10.1016/j. neucli.2013.10.138

3. Lacour M, Helmchen C, Vidall PP. Vestibular compensation: the neuro-otologist's best friend. J Neurol. 2016; 263: 54-64. https://doi org/10.1007/s00415-015-7903-4.

4. Dunlap PM, Holmberg JM, Whitney SL. Vestibular rehabilitation advances in peripheral and central disorders. Curr Opin Neurol. 2019 Feb; 32(1): 137-144. https://doi.org/10.1097/WCO.0000000000000632

5. Jadanowski K, Budrewicz D, Koziorowska-Gawron E. [Eye movement disorders in diseases of the central nervous system] Department and Clinic of Neurology, Medical University of Wrocław 2010.

6. Arnold SA, Stewart AM, Moor HM, et al. The effectivness of vestibular Rehabilitation. Interversion of the Treating unilateral peripheral vestibular disorders. A sistematic reviev. Physiother Res Int. 2017 Jul; 22(3). https://doi.org/10.1002/pri.1635

7. Sowula K, Składzień J, Szaleniec J, et al. Otolaryngological symptoms in patients treated for tick- born diseases. Otolaryngol Pol. 2018 Feb 28 72(1): 30-34. https://doi.org/10.5604/01.3001.0011.5948

8. Ogrinc K, Maraspin V. Nervous System Involvement in Lyme Borreliosis. TODJ 2016 March 28; 10: 44-54. https://doi. org/10.2174/1874372201610010044
9. Garcia-Monco J C, Benach J L. Lyme Neuroborreliosis: Clinical Outcomes, Controversy, Pathogenesis, and Polymicrobial Infections. Ann Neurol. 2019 Jan; 85(1): 21-31. https://doi.org/10.1002/ana.25389 10. Chomel B. Lyme disease. Rev Sci Tech. 2015 Aug; 34(2): 569-76. https:// doi.org/10.20506/rst.34.2.2380

11. Izac JR, Marconi RT. Diversity of the Lyme disease of the spirochetes and its influence of immune response of infection and vaccination. Vet Clin North Am Small Anim Pract. 2019 Jul; 49(4): 671-686. https://doi. org/10.1016/j.cvsm.2019.02.007

12. Younger DS. Epidemiology of Lyme Neuroborreliosis. Neurol Clin. 2016 Nov; 34(4): 875-886. https://doi.org/10.1016/j.ncl.2016.05.005

13. Halperin JJ. Lyme neuroborreliosis. Curr Opin Infect Dis. 2019 Jun; 32(3): 259-264. https://doi.org/ 10.1097/QCO.0000000000000545

14. Rauer S, Kastenbauer S, Fingerle V, et al. Lyme Neuroborreliosis. Dtsch Arztebl Int. 2018 Nov 9; 115(45): 751-756. https://doi.org/10.3238/ arztebl.2018.0751

15. Jozefowicz-Korczynska M, Zamyslowska-Szmytke E, Piekarska A, et al. Vertigo and Sever Balance Instability as Symptoms of Lyme DiseaseLiterature Review and Case Report. Front Neuro. 2019 Nov 12; 10: 1172. https://doi.org/10.3389/fneur.2019.01172

16. Han BJ, Song HS, Soo Kimc JS. Vestibular Rehabilitation Therapy: Review of Indications, Mechanisms, and Key Exercises. J Clin Neurol. 2011 Dec; 7(4): 184-196. https://doi.org/10.3988/jcn.2011.7.4.184

17. Lacour M, Tighilet B. Plastic events in the vestibular nuclei during vestibular compensation: the brain orchestration of a "deafferentation" code. Restor Neurol Neurosci. 2010; 28(1): 19-35. https://doi.org/10.3233/ RNN-2010-0509

18. Lacour M, Bernard-Demanze L. Interaction between Vestibular Compensation Mechanisms and Vestibular Rehabilitation Therapy: 10 Recommendations for Optimal Functional Recovery. Front Neurol. 2014; 5: 285. https://doi.org/10.3389/fneur.2014.00285 